\title{
Objetos de Aprendizaje Accesibles: Análisis desde sus metadatos y su relación con las normas ecuatorianas NTE ISO IEC 40500 y 24751
}

\section{Determination Of Coefficient Convective Heat Transfer In The Blanching Process Of "Castilla" Blackberry (Rubus Glaucus Benth) Using The Method Of Finite Elements}

\author{
Paola Cristina Ingavelez-Guerra 1,2, José Ramón Hilera-González², Salvador Otón-Tortosa 2.
}

Recibido para publicación: 12 de julio de 2018 - Aceptado para publicación: 19 de agosto 2018

\section{RESUMEN}

El presente documento constituye un estudio de la accesibilidad de objetos de aprendizaje acorde a las normativas WCAG 2.0 y sus metadatos de accesibilidad para lo cual se emplea una muestra de 15 objetos de aprendizaje realizados por diferentes instituciones de educación superior del Ecuador. Se considera la importancia del empleo de metadatos de accesibilidad en los recursos digitales educativos para su posterior información idónea en necesidades y preferencias de posibles usuarios en situación de discapacidad. Se presenta la evaluación comparativa de la muestra y se determina su relación de cumplimiento de la Norma Técnica Ecuatoriana 40500 (WCAG 2.0).

PALABRAS CLAVE: Accesibilidad, metadatos, WCAG 2.0, objetos de aprendizaje, e-learning.

\section{ABSTRACT}

This document is a study of the accessibility of learning objects according to WCAG 2.0 regulations and its accessibility metadata for which a sample of 15 learning objects made by different university in Ecuador. The importance of the use of accessibility metadata in educational digital resources is considered for its subsequent appropriate information on the needs and preferences of potential users in a situation of disability. The comparative evaluation of the sample is presented and its relation of compliance with the Ecuadorian Technical Standard 40500 (WCAG 2.0).

KEYWORDS: Accessibility, metadata, WCAG 2.0, e-learning, disability

\footnotetext{
${ }^{1}$ Universidad Politécnica Salesiana, Ecuador. ${ }^{2}$ Universidad de Alcalá, España . E-mail: pcingavelez@ups.edu.ec; jose.hilera@uah.es, salvador.oton@uah.es
} 
Ingeniería e Innovación - Vol. 4:(2) Julio - Diciembre de 2018 (7 - 12)

\section{INTRODUCCIÓN}

La accesibilidad va más allá de un concepto, método o proceso asociado a discapacidad. Es un sentir de igualdad de condiciones en la participación y pertenencia de todos a un entorno común. Su relación con usabilidad y diseño universal marcan un completo uso de la creatividad humana a la hora de comprender la totalidad del concepto. La definición más completa [1] la describe como: "...la condición que deben cumplir los entornos, procesos, bienes, productos y servicios, así como los objetos o instrumentos, herramientas y dispositivos, para ser comprensibles, utilizables y practicables por todas las personas en condiciones de seguridad y comodidad y de la forma más autónoma y natural posible"

La ONU en la Convención sobre los derechos humanos y su Protocolo Facultativo [2] señala en su artículo 24 : "Los Estados Partes reconocen el derecho de las personas con discapacidad a la educación. Con miras a hacer efectivo este derecho sin discriminación y sobre la base de la igualdad de oportunidades, los Estados Partes asegurarán un sistema de educación inclusivo a todos los niveles así como la enseñanza a lo largo de la vida..." La UNESCO en la declaración de París 2012, recomienda a los estados miembros "promover la calidad aseguramiento y revisión por pares de los REA (Recursos Educativos Abiertos). Fomentar el desarrollo de mecanismos para la evaluación y certificación de los resultados de aprendizaje obtenidos a través de los REA." El foro mundial sobre la educación 2015 en su Declaración de Incheon Educación 2030 [3] señala que "Es preciso aprovechar las tecnologías de la información y la comunicación (TIC) para reforzar los sistemas educativos, la difusión de conocimientos, el acceso a la información, el aprendizaje efectivo y de calidad, y una prestación más eficaz de servicios".

En el tema de educación y discapacidad varios han sido los avances alcanzados, pero aún es difícil evaluar la acertada aplicación de ambientes virtuales accesibles. En muchos casos se particulariza el entorno virtual a una discapacidad específica, generando nuevas barreras de accesibilidad para otras patologías. [4]

Investigar sobre tecnologías de aprendizaje para estudiantes con discapacidad en el
Ecuador, demanda constantemente la búsqueda de investigaciones actualizadas que vayan acorde a una realidad cambiante. En el tema de discapacidad es importante considerar las cifras a nivel mundial y su tendencia, pues alrededor de 1000 millones de habitantes, o el 15 $\%$ de la población mundial, tienen algún tipo de discapacidad, y su incidencia es mayor en países en desarrollo, [5] lo que constituye información poco alentadora, puesto que actualmente las estructuras para el cuidado de la salud, la rehabilitación y la educación especial no alcanzan su pleno desarrollo.

En el Ecuador se tiene una población de 16.703.004 [5] de los cuales 433.169 tienen discapacidad, registradas en CONADIS de la siguiente manera: 46,68\% corresponde a física, $22,64 \%$ a intelectual, $12,88 \%$ a auditiva, $11.89 \%$ a visual, $4,71 \%$ a intelectual, 1,30\% lenguaje. El $63.1 \%$ corresponde a un grupo etario entre 18 a 65 años [6].

En cuanto a la legislación ecuatoriana varias normas relacionadas con discapacidad se encuentran vigentes, entre ellas se dispone de: NTE INEN ISO/IEC 40500 Accesibilidad a contenidos web, NTE INEN ISO/IEC 24751 Adaptabilidad y accesibilidad individualizadas en aprendizaje electrónico, educación y formación, NTE INEN ISO/IEC 29138 Consideraciones de accesibilidad para personas con discapacidad. El Reglamento de Ley Orgánica de discapacidades en su Capítulo III: Derechos de la personas con discapacidad, señala en su artículo 10 y 11 la obligatoriedad de contar con equipos multidisciplinarios en educación así como también el respeto a porcentajes de becas en la educación superior y su proceso de escolaridad [7].

En la actualidad, los países enfrentan el desafío de brindar una educación de calidad para todos, fortaleciendo el enfoque de la inclusión que poco a poco va ganando terreno en los ámbitos educativo y social, haciendo frente a los altos índices de exclusión, discriminación y desigualdad educativa. La creación de las condiciones para el desarrollo de la educación para todos, que garantice calidad con equidad, implica transformaciones en el sistema educativo, en sus culturas, políticas y prácticas, involucrando de manera activa y participativa procesos evaluativos que validen los esfuerzos realizados. Con estos antecedentes, el presente documento presenta un estudio de los estándares en 
metadatos planteados y normas de accesibilidad web propuestas. Se plantea la metodología de evaluación empleada con la descripción de sus componentes, y finalmente se describe un análisis comparativo de los objetos de aprendizaje con las normas existentes, determinando sus respectivas conclusiones y trabajo futuro.

\section{METODOLOGÍA}

Bajo el termino de accesibilidad encontramos varios conceptos relacionados tales como: flexibilidad, personalización, universalidad, usabilidad, interoperabilidad, reusabilidad, navegabilidad, adaptabilidad, entre otras, sin embargo es importante considerar que la adaptabilidad y la accesibilidad son dos terminologías que convergen cuando se trata de atender a la diversidad propia del ser humano (adaptabilidad), buscando proporcionar flexibilidad en su entorno (accesibilidad), de tal manera que permita acomodarse a las necesidades de cada usuario y sus preferencias

- Tecnología de Asistencia / Tecnologías emergentes/Rampas Digitales: Varios productos informáticos tanto en hardware como en software se han creado como herramientas de apoyo que faciliten la accesibilidad, así se dispone de lectores de pantalla, magnificadores de pantalla, programas de reconocimiento de voz, teclados adaptados, mouse apuntadores y de entrada de información entre otros [9].

- Objetos de Aprendizaje/Recursos Digitales: Dado que el proceso de enseñanza aprendizaje va más allá del uso tecnológico de herramientas, se requiere entender la sinergia que debe existir entre la tecnología y el diseño metodológico para establecer una propuesta coherente, considerando la computación ubicua y su relación con muchos dispositivos y sistemas simultáneos. Los objetos de aprendizaje, podría entenderse como cualquier recurso digital multimedia empleado en ambientes virtuales de aprendizaje, cuya finalidad es integrar un acervo compartible en un ambiente educacional, por lo que deben cumplir con determinadas características que faciliten su reutilización interoperabilidad y accesibilidad [10].

- Universal Design for Learnig - UDL: Los esfuerzos metodológicos que emplea la corriente de Diseño Universal del Aprendizaje - DUA entiende el reto de la diversidad de aprendizaje de los estudiantes y potencializa la construcción de materiales flexibles, técnicas y estrategias orientadas a un mayor número de usuarios, proporcionando múltiples medios de representación, expresión y compromiso [8]

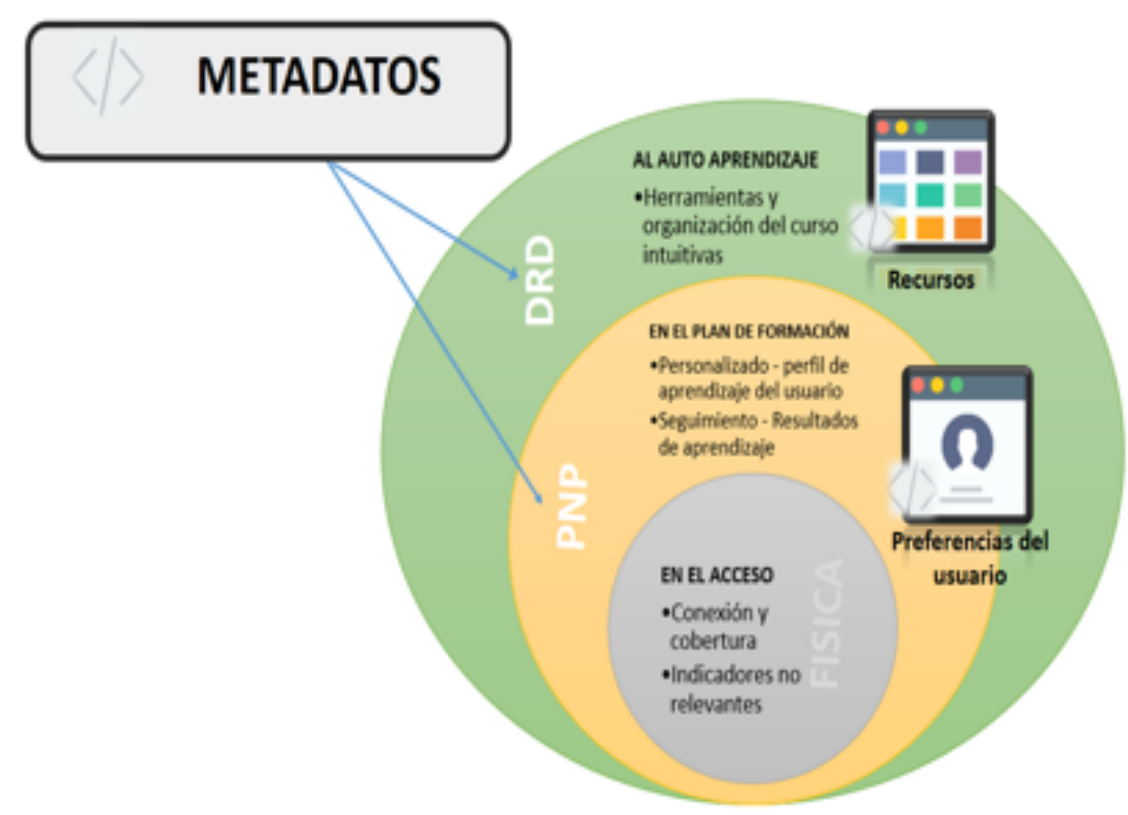

Figura 1. Metadatos de Accesibilidad 
- Metadatos: Para la descripción de las características de accesibilidad de los contenidos publicados en objetos de aprendizaje, es necesario utilizar mecanismos de descripción de información basado en metadatos, lo que facilitaría la información de un recurso digital y su posible requerimiento en base a preferencias y necesidades del estudiante. Se considera como referente en metadatos frecuentemente empleados a Schema.org [10]. Los metadatos de accesibilidad que define Schema.org están basados en IMS AfA v3.0 [11] que responde al estándar ISO/IEC 24751 Adaptabilidad y accesibilidad individualizadas en aprendizaje electrónico, educación y formación. En el caso de estudiantes con discapacidad, dicha información podría ser muy relevante, pues contiene información importante para su interacción. Los metadatos de accesibilidad permiten describir las características de accesibilidad del recurso (DRD) y/o de las preferencias y necesidades del ususario (PNP) como se muestra en la Figura 1.
La propuesta de metadatos de accesibilidad constituye información relevante para describir cualquier contenido web de una manera sencilla. [11].

\section{PROPUESTA METODOLÓGICA}

Se plantea una propuesta metodológica para evaluar objetos de aprendizaje acorde a la normativa ISO 40500, considerando que los objetos de aprendizaje dentro de un entorno virtual, responde a un contenido web, por lo tanto debe cumplir los estándares y directrices de la WCAG 2.0 .

Paralelamente existen los metadatos de accesibilidad especificados por Schema.org, como la descripción de la información que posee el objeto de aprendizaje. En caso de estudiantes con discapacidad, ésta información resulta imprescindible para optar por un recurso específico que responda a su necesidad y/o preferencia.

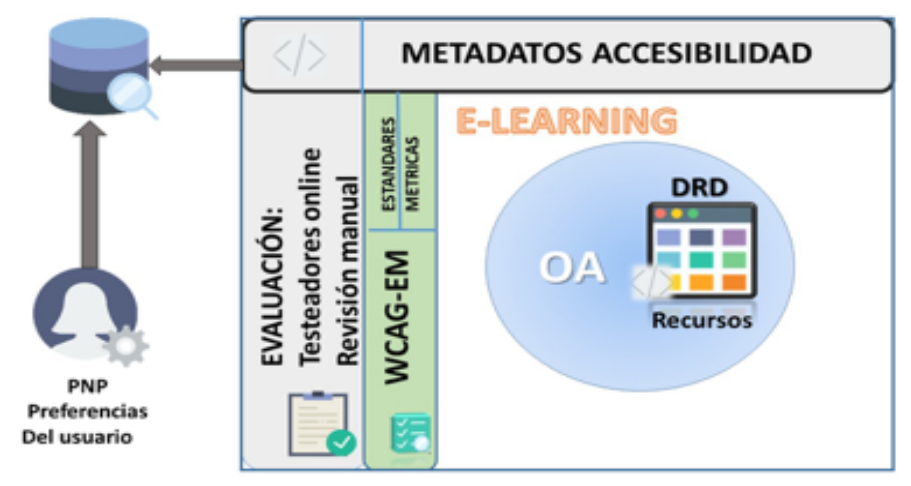

Metadatos de Accesibilidad: Se capturan los metadatos de accesibilidad establecidos en los recursos digitales educativos, en su gran mayoría realizados con ExeLearning. Cabe señalar que actualmente la herramienta no cuenta con metadatos de accesibilidad por lo que se procede a realizar un análisis para incorporación de metadatos.

Evaluación: Se realiza un análisis de los 53 criterios de conformidad en su nivel A y AA con el apoyo de herramientas on line y revisión manual con simuladores de discapacidad y se considera la Metodología de Evaluación de Conformidad con la Accesibilidad en sitios Web (WCAG-EM). Se establece un estudio de tipo cuasi-experimental con una sola muestra conformada por 15 OA (Objeto de Aprendizaje) que contienen 57 páginas html previamente seleccionadas acorde al tipo de OA y sugerencias de la Metodología empleada.

Recursos y preferencias: Se determinan características de accesibilidad de objetos de aprendizaje según WCAG y metadatos de accesibilidad. Se establecen relaciones con 18 metadatos de accesibilidad frecuentemente empleados.

\section{DISCUSIÓN DE RESULTADOS OBTENIDOS}

En la fase de evaluación se considera como relevantes las páginas web de tipo: index, contenido, páginas con incrustación de video/ audio o libro, y autoevaluación como se muestra en la Figura 3: 


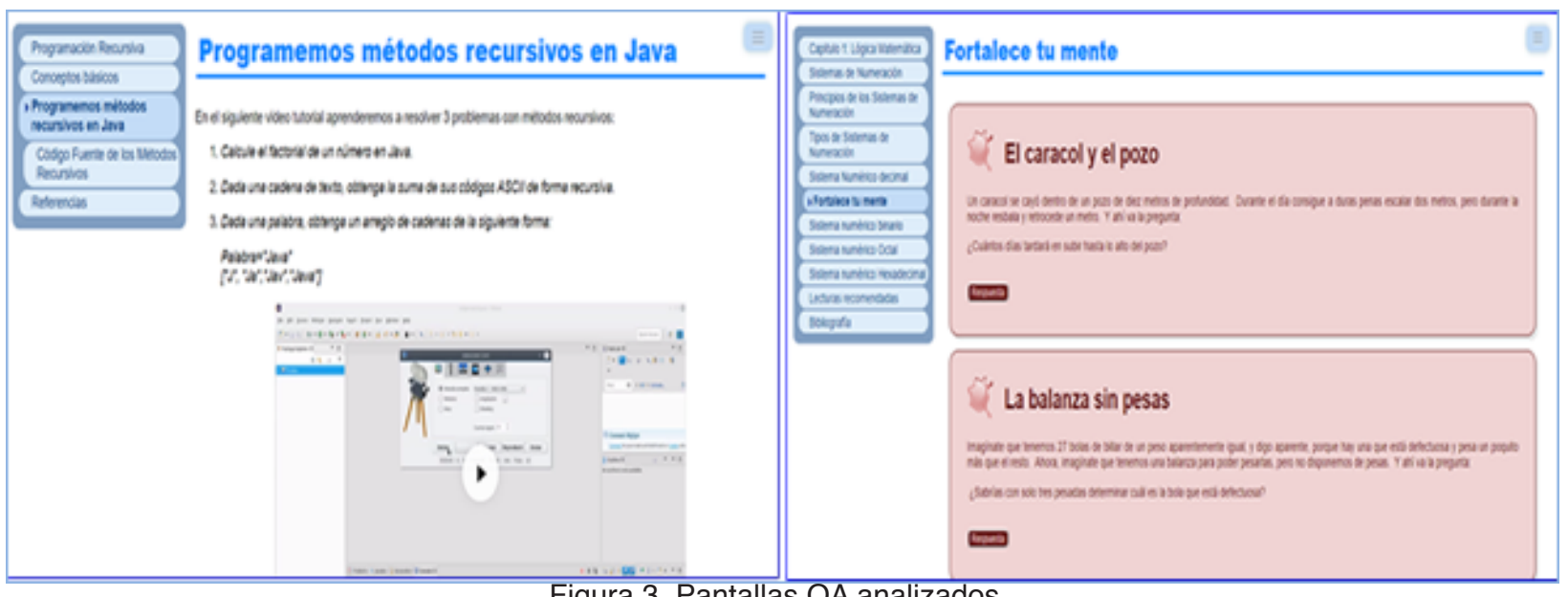

Figura 3. Pantallas $\mathrm{OA}$ analizados

Con los OA seleccionados se analiza la con TENON.IO y AXE. Los resultados se reflejan accesibilidad con 2 herramientas online : en la siguiente Figura:

Examinator y OAW, confirmando validaciones

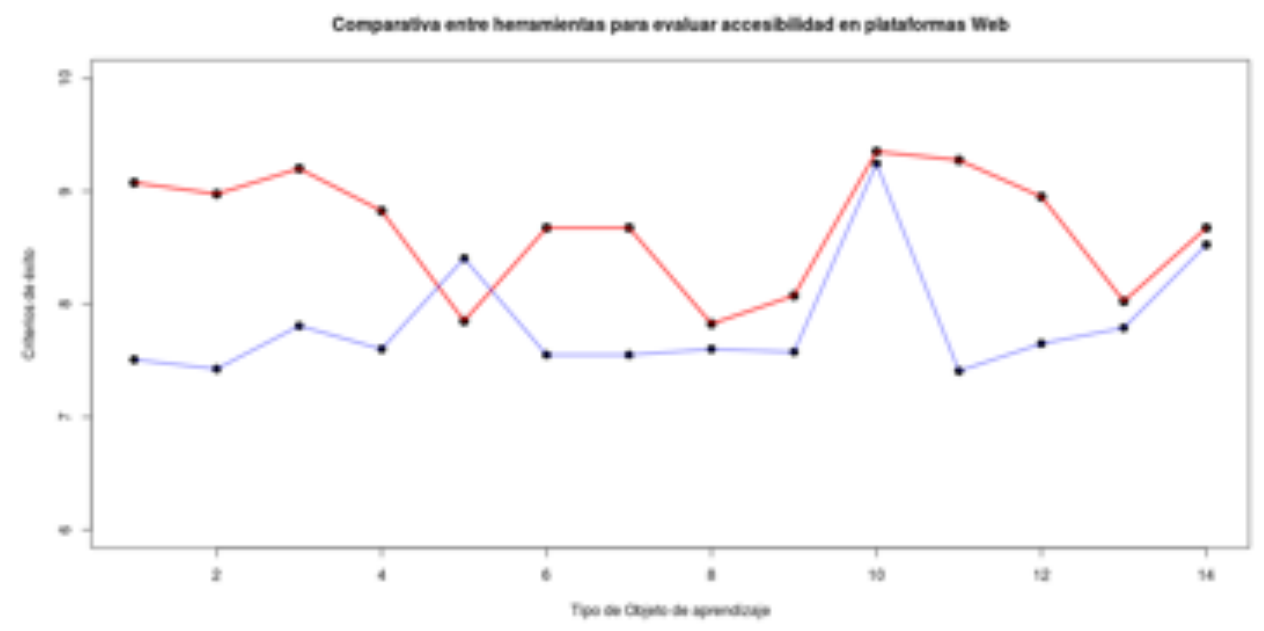

Figura 4. Comparativa de evaluación de accesibilidad web

La media de los $15 \mathrm{OA}$ es de 8,2/10. Se determina como mínimo 7,7 que responde a un $\mathrm{OA}$ empaquetado con animación flash con única página HTML, y una máxima de 9,29 que involucra en su mayoría a paginas bien formadas con incrustaciones propias de videos y sin frames ni cuestionarios de autoevaluación.

Los problemas detectados con mayor incidencia son identificados en la siguiente tabla:

Tabla 2. Errores Frecuentes

\begin{tabular}{|l|r|}
\hline DETALLE & INCIDENCIA \\
\hline Texto justificado & 49 \\
\hline Falta de atributos title & 45 \\
\hline
\end{tabular}

\begin{tabular}{|l|r|}
\hline iframe sin title & 45 \\
\hline Etiquetas alt invalidas & 30 \\
\hline $\begin{array}{l}\text { uso de medidas absolutas en } \\
\text { fuentes }\end{array}$ & 30 \\
\hline imágenes con atributo alt vacio & 19 \\
\hline tabla sin celda de encabezado & 16 \\
\hline formulario sin botón & 30 \\
\hline $\begin{array}{l}\text { CSS sin color primer plano y } \\
\text { fondo }\end{array}$ & 10 \\
\hline $\begin{array}{l}\text { enlaces con el mismo texto y } \\
\text { diferente destino }\end{array}$ & 12 \\
\hline $\begin{array}{l}\text { atributos alt para controlar pre- } \\
\text { sentación de texto }\end{array}$ & 2 \\
\hline
\end{tabular}


Ingeniería e Innovación - Vol. 4:(2) Julio - Diciembre de 2018 (7 - 12)

imágenes con más de 100 car-

acteres en atributo alt

El texto justificado, uso de fuentes absolutas, iframe sin títulos y el incorrecto uso de etiquetas alt, constituyen los principales errores.

Como aciertos en accesibilidad y su incidencia en los $\mathrm{OA}$ analizados encontramos:

Tabla 3. Critetrios de Éxito

\begin{tabular}{|l|l|}
\hline CRITERIOS DE ÉXITO & INCIDENCIAS \\
\hline $\begin{array}{l}\text { El primer enlace de la página lleva } \\
\text { al contenido principal de la página }\end{array}$ & 57 \\
\hline $\begin{array}{l}\text { Hay un enlace que permite saltar } \\
\text { bloques de contenido }\end{array}$ & 57 \\
\hline $\begin{array}{l}\text { No se usan elementos para con- } \\
\text { trolar la presentación visual }\end{array}$ & 57 \\
\hline $\begin{array}{l}\text { Se identifica el idioma principal de } \\
\text { la página con el codigo "es" /"en" }\end{array}$ & 58 \\
\hline La página tiene un element title & 53 \\
\hline $\begin{array}{l}\text { Todos los controles de formulario } \\
\text { tienen una etiqueta asociada }\end{array}$ & 6 \\
\hline $\begin{array}{l}\text { Todos los formularios tienen un } \\
\text { botón de envío }\end{array}$ & 1 \\
\hline $\begin{array}{l}\text { Todas las medidas en las CSS } \\
\text { están expresadas con valores } \\
\text { relativos }\end{array}$ & 4 \\
\hline $\begin{array}{l}\text { Todas las medidas en los atributos } \\
\text { HTML están expresadas en va- } \\
\text { lores relativos }\end{array}$ & 1 \\
\hline
\end{tabular}

Con relación a los metadatos detectados en los OA se establece una incidencia mayor en los siguientes metadatos:

Tabla 4. Metadatos Frecuentes

\begin{tabular}{|l|r|}
\hline METADATO & INCIDENCIA \\
\hline Modo de acceso Auditivo & 17 \\
\hline Modo de acceso TACTIL & 55 \\
\hline Modo de acceso TEXTUAL & 55 \\
\hline Modo de acceso VISUAL & 38 \\
\hline $\begin{array}{l}\text { Recurso está formateado, para } \\
\text { lectura de letra grande? }\end{array}$ & 48 \\
\hline $\begin{array}{l}\text { Dispone de una estructura básica o } \\
\text { tabla de contenidos? }\end{array}$ & 56 \\
\hline
\end{tabular}

\begin{tabular}{|l|r|}
\hline $\begin{array}{l}\text { Los videos empleados tienen sub- } \\
\text { titulado? }\end{array}$ & 10 \\
\hline $\begin{array}{l}\text { La navegación en el objeto de } \\
\text { aprendizaje tiene una estructura } \\
\text { organizada e intuitiva? }\end{array}$ & 53 \\
\hline $\begin{array}{l}\text { El usuario puede acceder y contro- } \\
\text { lar el contenido de forma efectiva } \\
\text { solo con el teclado? }\end{array}$ & 53 \\
\hline $\begin{array}{l}\text { El usuario puede acceder y contro- } \\
\text { lar contenido de manera efectiva } \\
\text { solo con el mouse? }\end{array}$ & 56 \\
\hline
\end{tabular}

Se destacan las características de modos de acceso Textual y Táctil en la mayoría, su control total con teclado y una estructura de navegación coherente propia de la herramienta ExeLearning para crear los OA.

\section{DISCUSIÓN DE RESULTADOS OBTENIDOS}

Es necesario establecer herramientas que faciliten la incorporación de código asociado a metadatos de accesibilidad en objetos de aprendizaje, acorde a características detectables y de manera automática. El desarrollar un objeto de aprendizaje por parte de docentes, requiere de destrezas tecnológicas y manejo de recursos multimediales, por lo que, si adicionamos la carga de conocimiento asociado a metadatos, la complejidad sería mayor

Existen varios testeadores online de accesibilidad óptimos para el desarrollo y corrección de páginas web y que son vistos desde el perfil del programador, pero el desarrollo de contenido web, actualmente no es de uso exclusivo de un profesional informático, por lo que es necesario un estudio constante de herramientas generadoras que fortalezcan la accesibilidad y empleo de buenas prácticas de manera intuitiva.

En junio de 2018 se aprobó WCAG 2.1, por lo que para investigaciones futuras será necesario complementar los nuevos criterios de conformidad añadidos en la versión 2.1 que es compatible con la 2.0, ya que mantiene los 61 criterios de WCAG 2.0 y le añade 17 nuevos criterios [13] Seguramente las herramientas de evaluación automática deberán adaptarse también a la versión 2.1 lo antes posible. 


\section{REFERENCIAS}

[1]. Akterian, S. 1995. Numerical simulation of unsteady heat transfer in canned mushrooms in brine during sterilization process. Journal of Food Engineering, Vol 25. 45-53p.

[2]. Astrom, A. Bark, G. 1994. Heat transfer between fluid and particles in aseptic processing. Journal Food Engineering. Vol. 21. $97-125 p$.

[3]. Choi, Y. y Okos, M. 1986. Effects of Temperature and Composition on the Thermal Properties of Foods. Journal of Food Process and Applications 1(1), 93101.

[4]. Duarte, P. y Cristianini, M. 2011. Determining the Convective Heat Transfer Coefficient (h) in Thermal Process of Foods. International Journal of Food Engineering 7(4), art. 15.

[5]. Incropera, F.P. y DeWitt, D.P. 2007. Fundamentals of Heat and Mass Transfer. Sexta edición. Editorial J. Wiley \& Sons, New York.

[6]. Iribe-Salazar, R., Caro-Corrales, J., Hernández-Calderón, O., Zazueta-Niebla, J., Gutiérrez-Dorado, R., CarrazcoEscalante, M. y Vázquez-López, Y. 2015. Heat Transfer during Blanching and Hydrocooling of Broccoli Florets. Journal of Food Science 80(12), 2774-2781.

[7]. Jaiswal, A., Gupta, S. y Abu-Ghannam, N. 2012. Kinetic evaluation of colour, texture, polyphenols and antioxidant capacity of Irish York cabbage after blanching treatment. Food Chemistry 131(1), 63-72

[8]. Jeevitha, G., Hebbar, H. y Raghavarao, K. 2015. Modeling of Peroxidase Inactivation and Temperature Profile during Infrared Blanching of Red Bell Pepper. Journal of Food Processing and Preservation 40(1), 83-93.

[9]. INCAP. 2012. Tabla de Composición de
Alimentos de Centroamérica. Ed. INCAP/ OPS. Guatemala.

[10]. Mazo, J. Rodriguez, D. Echeverri, C. 1998. El cultivo de la mora (Rubus glaucus Benth): Principios agroecológicos para su manejo. Servicio Nacional de Aprendizaje, SENA. Antioquia. Colombia.

[11]. Mendoza, R. Herrera, A. 2012. Cinética de inactivación de la enzima peroxidasa, color y textura en papa criolla (Solanum tuberosum grupo phureja) sometida a tres condiciones de escaldado. Revista Información Tecnológica. vol 23 n 4. 73$82 \mathrm{p}$

[12]. Ordoñez, J. Morales, E. 1996. Determinación y evaluación del coeficiente convectivo de transferencia de calor en procesos continuos de escaldado e hidroenfriado. Tesis para optar por el título de Licenciado en Ingeniería de Alimentos. Valdivia. Universidad Austral de Chile. Facultad de Ciencias Agrarias. 154 p.

[13]. Sablani, S. S. 2009. Measurement of Surface Heat Transfer Coefficient, In Handbook of Food Properties. Second edition M. S. Rahman (Editor). Francis and Taylor Group. 697-716p. 